\title{
Mechanical Vibrations Applied to Nondestructive Evaluation of Materials and Structures
}

\author{
Luca Collini, ${ }^{1}$ Giada Gasparini, ${ }^{2}$ Michele Palermo, ${ }^{3}$ and Salvatore Russo ${ }^{4}$ \\ ${ }^{1}$ Department of Engineering and Architecture, University of Parma, 43124 Parma, Italy \\ ${ }^{2}$ Department of Civil, Chemical, Environmental, and Materials Engineering, University of Bologna, 40126 Bologna, Italy \\ ${ }^{3}$ Interdepartmental Centre for Industrial Research in Building and Construction, University of Bologna, 40126 Bologna, Italy \\ ${ }^{4}$ Iuav University of Venice, 0123 Venice, Italy \\ Correspondence should be addressed to Luca Collini; lucaferdinando.collini@gmail.com
}

Received 20 November 2017; Accepted 21 November 2017; Published 18 December 2017

Copyright (C) 2017 Luca Collini et al. This is an open access article distributed under the Creative Commons Attribution License, which permits unrestricted use, distribution, and reproduction in any medium, provided the original work is properly cited.

Since the first vibration-based damage detection technology in the monitoring of rotating machinery, up-to-date mechanical vibrations are successfully used in many other applications in the field of NDT and NDE, and we think that many open questions and possibilities are still left to be found. Vibrations are relatively simple measurable quantities, containing a set of information that is peculiar of the system we examine. Further, repeated measures, even after years, are precious witnesses of an evolving situation over the time for those ambits in which aging is critical as health and restoration problems in artistic heritage.

In this special issue devoted to applications of mechanical vibration methods in NDT and NDE, we tried to collect contributions from researchers and experts from the academic world and also belonging to the industrial world. The valuable contributions, 9 in number, have been selected in such a way that some of the most important themes of the main topic are touched, and even though ranging from industrial diagnostics to civil construction dynamical performance, in the whole they appear in a harmonious form.

The works are of high scientific level and introduce to the researches community of remarkable novelty. The issue contains a classical application of the frequency-based diagnosis method treated in the paper "The Absolute Deviation Rank Diagnostic Approach to Gear Tooth Composite Fault" by G. Wang et al. from the Hunan University of Science \& Technology of Xiangtan, China. In this work, the authors apply with success the absolute deviation rank approach for determining damage occurring in transmission gears, as teeth breakage or pitting. The frequency response of gears is shown to indicate different degrees and different types of damage.

Another contribution deals with the NDT methods for civil structures and is about the "Experimental Evaluation for the Microvibration Performance of a Segmented PC Method Based High Technology Industrial Facility Using 1/2 Scale Test Models" proposed by S. Kim and S. W. Choi from the Kyonggi University, Kyonggido, and the Catholic University of Daegu, Kyeongsan-si, both in Korea. In this work, the authors present an experimental work on a half-scale model of a bridge system built and assembled in segments to facilitate transportation due to the dimensions.

In the paper entitled "Experimental Research on Seismic Performance of a New-Type of R/C Beam-Column Joints with End-Plates" the authors S. Li et al. from the Xian University of Architecture and Technology, China, present the design of an end-plate for the connection of civil structures. Via a dynamical testing campaign, they calculate the dissipation of the joint that is a crucial parameter when in presence of seismic excitation.

Again from China, University of Shanghai for Science and Technology, there comes the research "Optimization Method of the Car Seat Rail Abnormal Noise Problem Based on the Finite Element Method" by H. Yu et al., in which experiments in conjunction with finite element analysis are used within the design of car seat supports; here stiffness and damping of the system are optimized for the comfort and safety. 
In another contribution to the special issue, D. Han et al. from the School of Vehicles and Energy, Yanshan University, Qinhuangdao (China), the School of Electrical Engineering, Yanshan University, Qinhuangdao (China), and the School of Electrical and Computer Engineering, Georgia Institute of Technology, Atlanta (USA), introduce a vibrationbased method for damage monitor with the paper "Damage Identification of Derrick Steel Structure Based on HHT Marginal Spectrum Amplitude Curvature Difference." In this interesting paper, the Hilbert-Huang transform marginal spectrum amplitude curvature is successfully applied for the damage sensitivity index to identify the damage in a derrick structure; the results show that the index can accurately determine the location of the damage element and weak damage element.

The paper "Vibration Analysis for Monitoring of Ancient Tie-Rods" by L. Collini et al. from the University of Parma (Italy) instead is devoted to the indirect monitoring of the axial load, which is commonly unknown especially after many years, in structural tie-rods placed as reinforcing elements in historical buildings. The problem of assessing the healthy condition of such elements is crucial for determining the building stability and a ND method based on the measure of vibrations looks very useful.

In the paper "Damage Identification of Unreinforced Masonry Panels Using Vibration-Based Techniques" by C. Oyarzo-Vera and Nawawi Chouw from the Universidad Católica de la Santísima Concepción, Concepción, Chile, and from the University of Auckland, New Zealand, the changing of modal parameters is analyzed to detect damage in a masonry specimen; the experimental method works properly, even if a large number of measurement points need to be considered to obtain an acceptable level of resolution.

One paper is entitled "A Signal Decomposition Method for Ultrasonic Guided Wave Generated from Debonding Combining Smoothed Pseudo Wigner-Ville Distribution and Vold-Kalman Filter Order Tracking" and is presented by J. Wu et al. from the Nanjing Forestry University, Nanjing, China. In this work, the wave packets of ultrasonic nondestructive evaluation and testing applied to carbon fiber composites are analyzed via a signal decomposition method in order to detect the debonding damage, which are characteristic of these materials, in terms of size and location.

Another paper, entitled "The Monitoring of Palazzo Lombardia in Milan" is presented by an Italian team of researchers from the Polytechnic of Milan and from the University of Parma, Italy, M. Berardengo et al., and deals with the estimation of modal parameters of Palazzo Lombardia, one of the tallest high-rise buildings in Italy, through operational modal analysis. The work conjugates an extensive experimental monitoring activity through transducers and accelerometers positioned ad hoc along the building, with a statistical analysis of data allowing the draft of a model for the relationships between eigenfrequencies and environmental variables aiming at a general structural health monitoring procedure which is based on the evolution of the building modal parameters.
Hoping the issue results are of interest for academic and technical readers, we wish a fruitful reading.

Luca Collini

Giada Gasparini

Michele Palermo

Salvatore Russo 


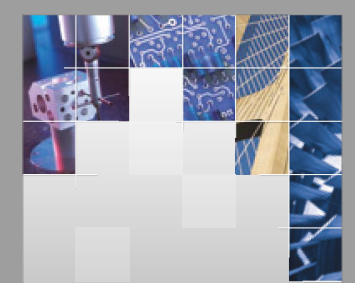

\section{Enfincering}
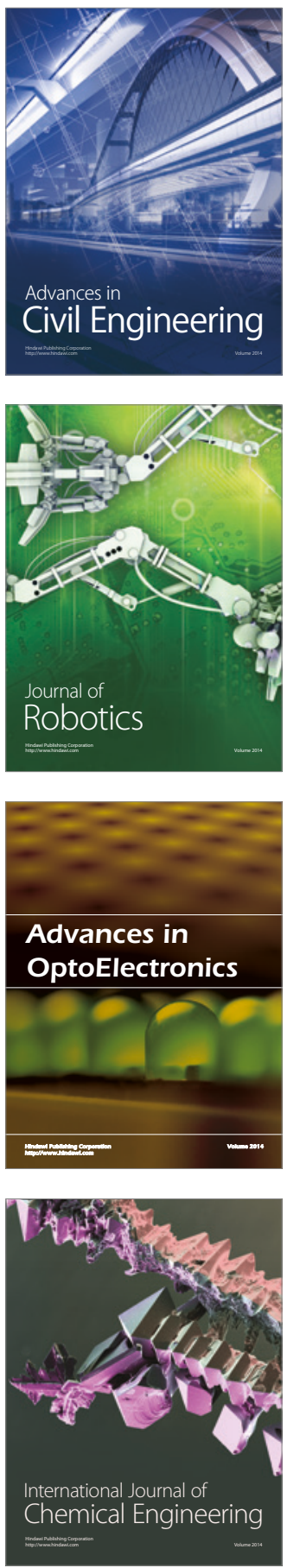

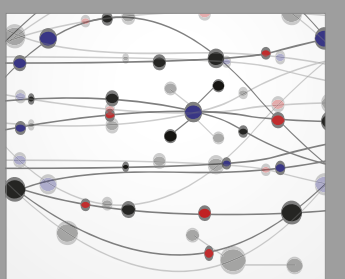

The Scientific World Journal

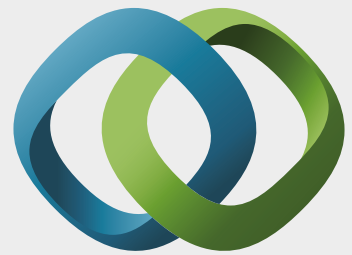

\section{Hindawi}

Submit your manuscripts at

https://www.hindawi.com
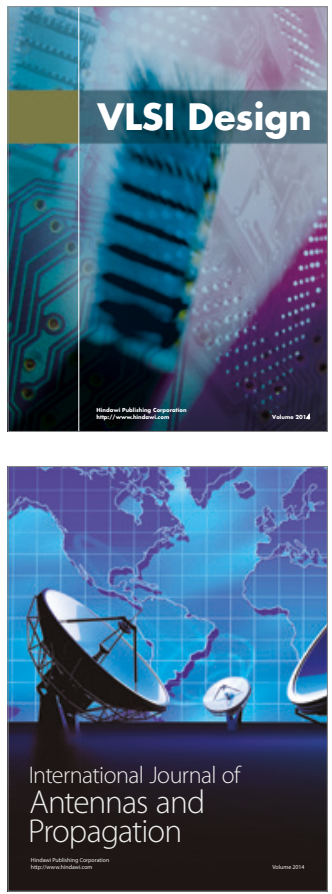

\section{Rotating}

Machinery
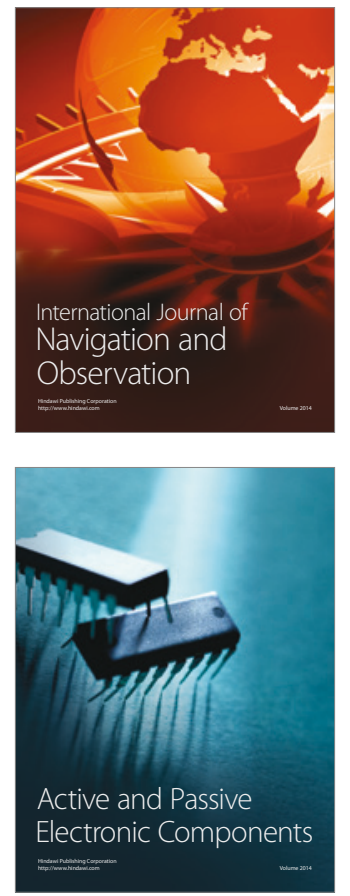
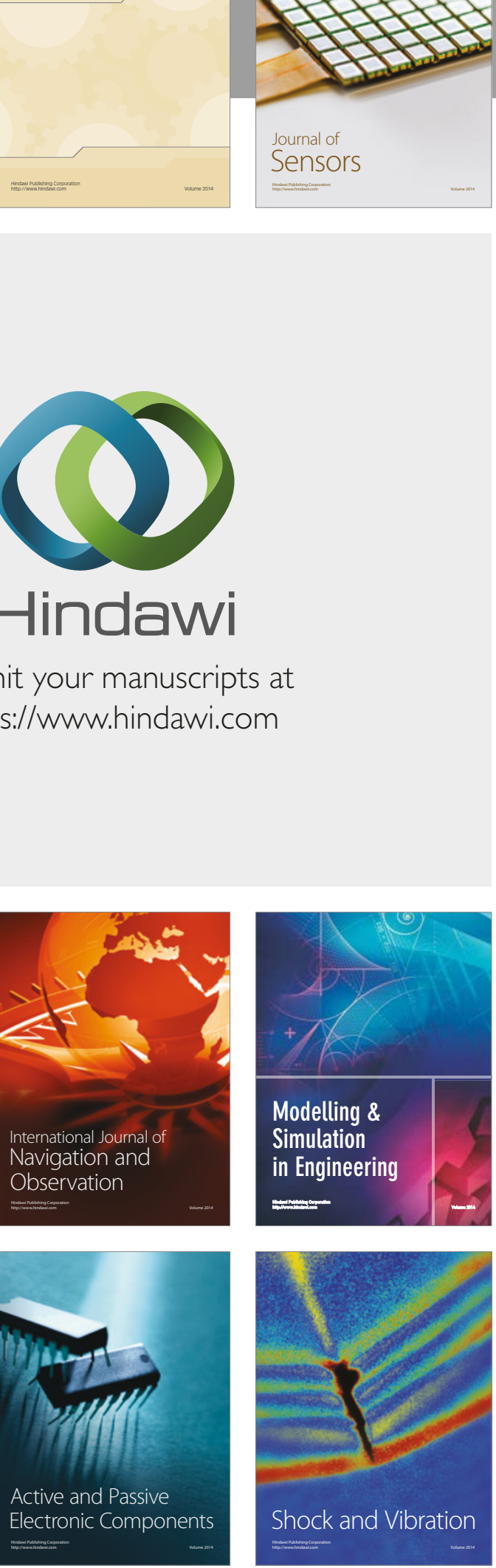
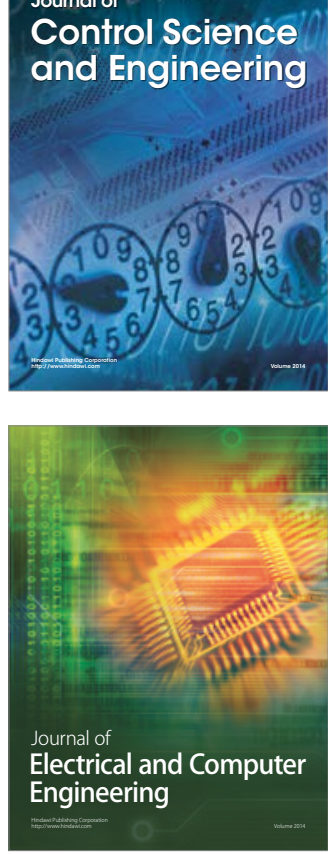

Distributed

Journal of

Control Science

and Engineering
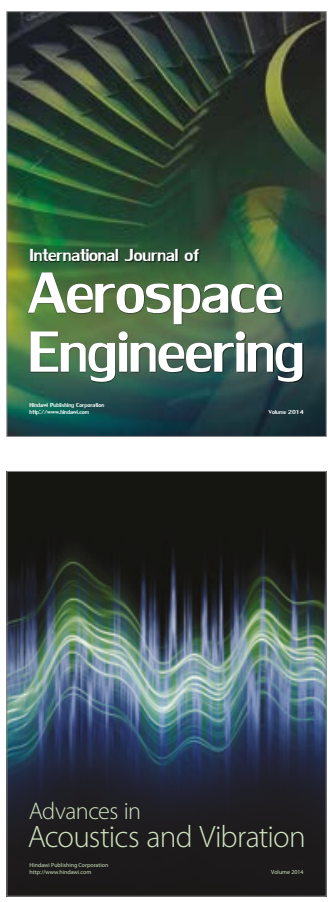

Sensor Networks 